\title{
Lima, Tiempo Social y Desplazamientos Motorizados
}

\author{
Lima, Social Time and Motorized Travel \\ Lima, Tempo Social e Viagem Motorizada
}

\author{
Zulema Conto Quispe. \\ Arquitecta, Msc en Gestión y Valoración Ph.D(c) Arquitectura, Edificación, Urbanística y Paisaje. \\ Universitat Politècnica de València, España. \\ zuconqui@doctor.upv.es \\ (iD) https://orcid.org/0000-0002-1971-7648
}

Recibido: diciembre 30 de 2019 Aceptado: febrero 13 de 2020 Publicado: febrero 20 de 2020

\section{RESUMEN}

A pesar de que, desde hace décadas, el uso generalizado del automóvil, como medio de transporte en las ciudades, ha sido cuestionado, y hoy muchas ciudades se enfocan en calmar el tráfico con políticas de restricción del uso del automóvil, en la ciudad de Lima el actual modelo de movilidad prioriza los modos motorizados privados, a pesar de que la mayoría de la población se desplaza en transporte público. El propósito de este artículo es evidenciar el tiempo social que pierden los limeños a causa del sistema de transporte, tanto si se desplazan en automóvil propio, como en transporte público.

Palabras clave: Movilidad urbana, tiempo social, desplazamientos motorizados, transporte público, Lima

\begin{abstract}
Although, for decades, the widespread use of the automobile, as a means of transportation in cities, has been questioned, and today many cities focus on calming traffic with policies restricting the use of the automobile, in the city of Lima the current mobility model prioritizes private motorized modes, despite the fact that the majority of the population travels by public transport. The purpose of this article is to show the social time that Lima's people lose because of the transport system, whether they travel by car or by public transport.
\end{abstract}

Keywords: Urban mobility, social time, motorized movements, public transport, Lima

\section{RESUMO}

Embora, por décadas, o uso generalizado do automóvel, como meio de transporte nas cidades, tenha sido questionado, e hoje muitas cidades se concentram em acalmar o tráfego com políticas que restringem o uso do automóvel, na cidade de Lima, o atual modelo de mobilidade prioriza os modos motorizados privados, apesar de a maioria da população viajar de transporte público. O objetivo deste artigo é mostrar o tempo social que as pessoas de Lima perdem por causa do sistema de transporte, viajando de carro ou de transporte público.

Palavras-chave: Mobilidade urbana, tempo social, movimentos motorizados, transporte público, Lima 


\section{INTRODUCCIÓN}

Desde hace décadas, varios autores han puesto en evidencia los contrasentidos y las irracionalidades a los que conduce el uso generalizado del automóvil, como medio de desplazamiento en las ciudades. Uno de los primeros en dar a conocer su preocupación por el incremento de los vehículos de motor fue Colin Buchanan, quien, en 1960, por encargo del Parlamento Británico, elaboró un estudio del desarrollo, a largo plazo, del tráfico de motor en las áreas urbanas, informe que publicó en el libro 'Traffic in Towns' (conocido como el Informe Buchanan). En este manifiesta que, a menos que se hiciera algo, el conflicto entre las ciudades en su forma presente y los vehículos de motor, tal como se utilizaban en ese momento, se iría haciendo progresivamente mayor. Por su parte, la urbanista y activista Jane Jacobs, en 1961, cambia la manera de ver la temática urbana, en su ya conocido libro 'The death and life of great american cities', en el que aborda las consecuencias de la erosión de las ciudades a causa de los automóviles.

En la misma década, Mishan (1969) habla de cómo el uso del automóvil crece a manera de espiral, y que, además, este repercute en el transporte público. Menciona que, a partir de un cierto nivel, cuanto más gastan los ciudadanos en automóviles para desplazarse, peor servicio les otorga este medio, llegando al punto en que pueden tardar mucho más en los desplazamientos de lo que tardaban antes de utilizarlo. Ejemplifica su razonamiento en tres fases: en la Fase l, en la cual no existe tráfico privado y un eficiente sistema de transporte público, el individuo A, utiliza diariamente el autobús que lo lleva al centro de la ciudad en unos diez minutos; en la Fase II, que es de transición, A compra un automóvil, con el cual espera que lo lleve al centro de la ciudad en cinco minutos; la Fase III aparece cuando un gran número de individuos sigue el ejemplo de $A, y$ al cabo de dos o tres años, se produce un incremento en el número de automóviles privados, por lo que A necesita quince minutos para llegar a su trabajo. Entonces, se da cuenta de que se hallaba en mejor situación en la Fase I, pero ya no tiene abierta esta oportunidad, puesto que, debido al aumento en el número de automóviles privados, la congestión del tráfico es tal, que necesitaría veinticinco minutos para llegar a su oficina si tomase el autobús.
Pero es Iván Ilich (1973) quien revoluciona la manera en el que se ve al automóvil. Asocia el transporte no solo con el tiempo que se requiere para conducirlo, sino también con el tiempo de trabajo que se requiere para su compra y mantenimiento. Realiza cálculos para un norteamericano promedio y cuánto tiempo este le dedica a su auto, evidenciando que la velocidad a la que va, es menor que la velocidad a la que va un ciclista. Bajo este mismo enfoque, Dupuy \& Robert (1979) se cuestionan: "¿Hace el automóvil ganar tiempo?" (p. 46); y Naredo \& Sánchez (1981) realizan las cuentas del automóvil desde el punto de vista del usuario.

A partir de estos aportes $-y$ bajo la misma metodología utilizada por los autores antes mencionados-, el objetivo de este artículo es evidenciar el 'tiempo social' que pierden los limeños en sus desplazamientos, tanto si se desplazan en auto propio como en transporte público, evidenciándose que estos desplazamientos implican un dispendio de su tiempo social.

\section{Lima, el Escenario Metropolitano}

El tipo de desarrollo que ha seguido el Perú, desde la época de la colonia hasta la actualidad, ha fortalecido el centralismo y la concentración de las actividades, tanto económicas, sociales, culturales, y político-administrativas en el ámbito territorial del Área Metropolitana de Lima (AML), situación que ha determinado la polarización del crecimiento urbano y demográfico frente al resto de ciudades. En este contexto, concentra más del 30\% de la población peruana. A partir de la segunda mitad del siglo $X X$, se produjo el crecimiento de la población y de la superficie, producto de la migración interna del campo a la ciudad. Todo ello dio lugar a una expansión urbana basada en el crecimiento periférico de baja densidad, configurando una "ciudad dispersa", donde los mayores niveles de densidad se localizan en los distritos de la zona central de la metrópoli, y se trasladan paulatinamente a los distritos periféricos (Figura 1). La extensión de la ciudad estableció un alejamiento entre determinados usos del suelo y funciones urbanas, $y$, por tanto, cada vez mayores distancias a recorrer, sobre todo para aquellos sectores que se ubican en las zonas periféricas. 

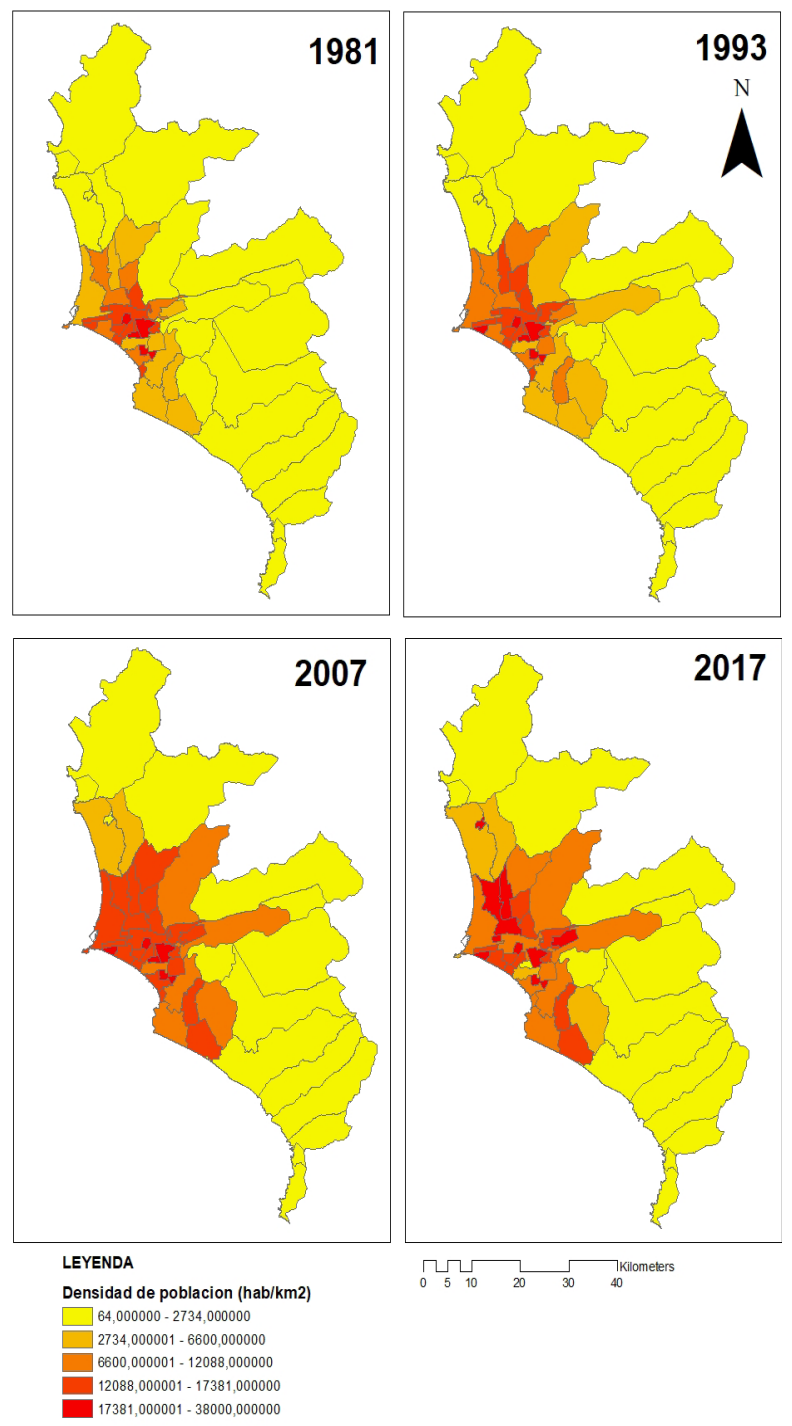

Figura 1. Variación de la Densidad 1981-2017.

Fuente: Elaboración Propia con base en INEI, Censos 1981, 1993, 2007, 2017.

Desde su fundación, la organización espacial de Lima estuvo basada en un solo centro. Durante siglos, el Centro Histórico de Lima cumplió funciones múltiples (sede de gobierno, administrativo, financiero, cultural, etc.). A partir de la explosión demográfica de 1940, se llega a configurar una estructura radial metropolitana articulada por este centro. A pesar de que en las últimas décadas, muchos investigadores como González \& Del Pozo (2012), Vega-Centeno (2017) y Fernández \& Vilela (2015) identifican nuevas centralidades en la metrópoli -que si bien le han otorgado mayor dinamismo económico y se constituyen como destino laboral para estos sectores-, aún desempeñan un rol secundario dentro de la estructura metropolitana.
A pesar de los débiles intentos de desconcentración de actividades urbanas, Lima mantiene una estructura centralizada-monocéntrica, que actúa como un imán que produce grandes desplazamientos motorizados, a la zona central desde las zonas periféricas. En la Figura 2, se observa la ubicación, tanto de las centralidades metropolitanas como de las locales.

Las centralidades metropolitanas están referidas a aquellas concentraciones urbanas que, indistintamente de su ubicación, tienen un alto nivel de atracción respecto al área metropolitana, además de estar asociadas con desplazamientos de distancias largas, por lo que depende de buenos enlaces de transporte, situación que actualmente no ocurre. Las centralidades y los centros especializados metropolitanos, según el PLAM (Plan Metropolitano de Desarrollo Urbano Lima y Callao 2035) son siete y los conforman: el Mercado Central y el Centro Comercial de Mesa Redonda; el Centro Comercial Industrial de Gamarra; el Centro Comercial de Miraflores; y el Centro Financiero de San Isidro. Estas cuatro centralidades constituyen las principales aglomeraciones, tanto por su extensión como por su nivel de concentración. A estos, se suman el Centro Comercial de San Miguel/Universitaria; Lince; y La Cultura. Las 7 concentraciones urbanas se localizan en el sector de Lima Centro.

Mientras que las centralidades locales se refieren a los establecimientos o concentraciones urbanas de escala local, y seasocian con los viajes locales cortoso cotidianos, estas concentraciones urbanas de menor escala las constituyen 31 centros y centralidades de jerarquía interdistrital y local. Mayoritariamente, se ubican en el sector de Lima Centro, seguido de Lima Norte en menor porcentajes.

Frente a esta configuración espacial, el actual modelo de movilidad urbana no solo prioriza los modos motorizados frente a los no motorizados, sino que da relevancia a la movilidad motorizada privada, curioso en una metrópoli donde la mayoría de su población se desplaza en transporte público, $73.4 \%$, según Lima Cómo Vamos al 2017. Dos aspectos han contribuido a definir su actual modelo de movilidad. Primero, el 'incentivo del crecimiento de unidades vehiculares motorizadas', que se da a partir de la década de los 90, con la liberalización del transporte urbano, a través del Decreto Legislativo 651. Este establecía la libre competencia de tarifas de servicio público de transporte, libre acceso a las rutas, la posibilidad de que cualquier persona natural o jurídica prestase servicio de transporte público, y el permiso para que cualquier vehículo (exceptuando camiones y vehículos de dos ruedas) pudiese brindar el servicio de transporte público. 

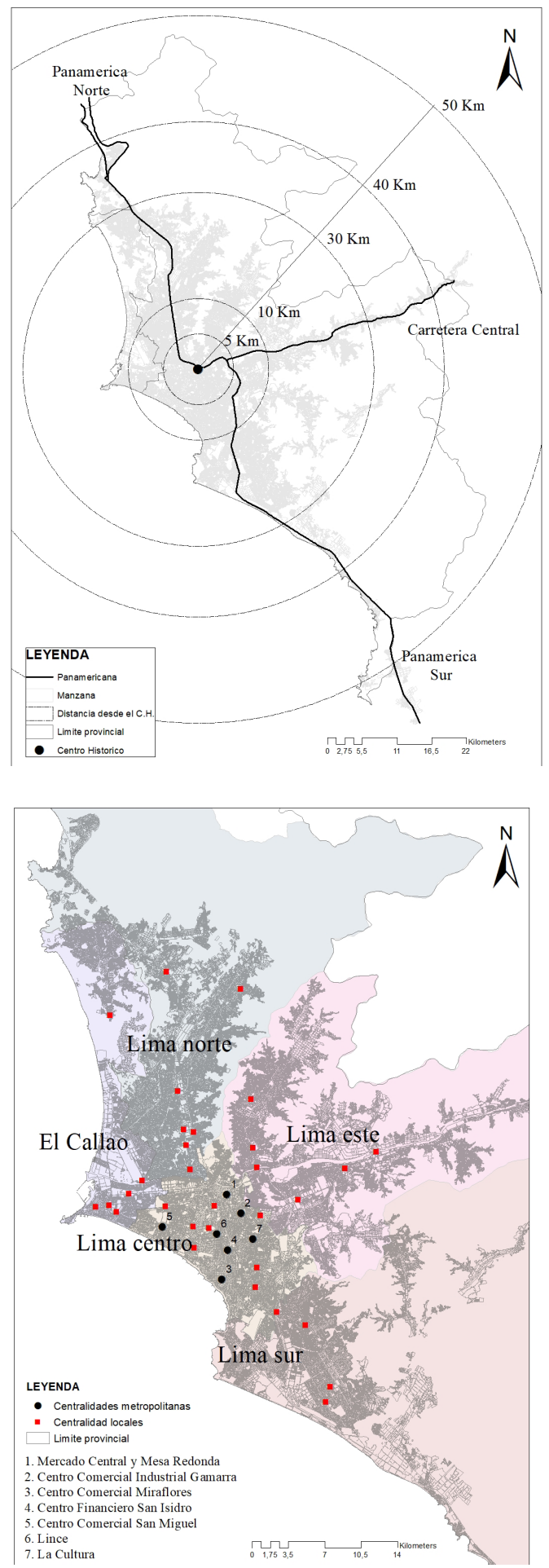

Figura 2. Ubicación de las Principales Centralidades Metropolitana y Locales.

Fuente: Elaboración Propia con base en Chión (2002), González \& Del Pozo (2012), Vega-Centeno (2015), Fernández \& Vilela (2015).

Segundo, el Decreto Supremo 080-91-EF, que eliminaba las restricciones de las importaciones y exportaciones de vehículos usados. Esta situación lejos de solucionar la crisis de la sobredemanda del transporte de ese entonces, se convirtió en una sobreoferta y en el crecimiento desmesurado del parque automotor, problemática que subsiste hasta la actualidad, y que va acompañado de la obsolescencia del parque vehicular, siendo la edad promedio del parque vehicular de servicio público de 22,5 años y el privado de 15,5 años, según Lima Como Vamos al 2017, además de la presencia de transporte informal. Se estima que el parque automotor, para el Departamento de Lima, es de $1,674,145$ (68.8\% del parque automotor nacional), y que, desde el año 2010 hasta el 2015, este se ha incrementado en un $40 \%$, lo que significa un aumento de 478,792 unidades de vehículos en 5 años, según el Ministerio de Transportes y Comunicaciones.

Un segundo factor, para esta priorización de la movilidad motorizada, es el 'enfoque de la demanda en el diseño de la infraestructura vial.' Este enfoque, según Herce (2009), consiste en poner el énfasis de análisis en la cuantificación de la solicitación futura de la red, ampliando o alterando las redes existentes con una clara preferencia de automóvil sobre el peatón. Este ha sido y sigue siendo el sustento en la intervención del sistema de transporte urbano en el AML, consecuencia, en parte, a como se han desarrollado y se siguen desarrollando las encuestas de transporte y movilidad, que se enfocan en identificar los denominados "motivos obligados" (trabajo y estudio), y preferentemente los modos de desplazamiento motorizado, dejando de lado aquellos que, hasta hace poco, no eran de interés en los estudios de movilidad, conocidos como "motivos no obligados" (ocio, hacer compras, llevar a los niños al colegio), o tomando en consideración los factores sociales, culturales, o cuestiones de género, edad, discapacidad, etc.

En términos generales, tanto la expansión periférica - que se traduce en cada vez mayores distancia a recorrer-, como el monocentrismo -que fomenta desplazamientos motorizados y el crecimiento de la unidades motorizadas-, están contribuyendo cada vez más a generar mayores índices de trafico motorizados y congestión vehicular, situación que repercute sobre el incremento del tiempo en los desplazamientos de las personas.

\section{Tiempo Social y Desplazamientos Motorizados}

Para hablar del tiempo dedicado a los desplazamientos motorizados, vamos a mencionar a Iván Illich (1974), cuyo mayor aporte, cuando se refiere al auto, es la manera de asociar el transporte no solo con el tiempo que requiere para conducirlo, 
sino también con el tiempo de trabajo que se requiere para su compra y mantenimiento, aporte conocido a través del siguiente párrafo:

El americano medio gasta más de mil seiscientas horas anuales en su coche, ya sea con este en movimiento o detenido. Aparca, o pretende hacerlo; gana dinero para invertirlo en él y pagar las cuotas mensuales; trabaja para pagar el combustible, los peajes, el seguro, los impuestos y las multas. Así, consume cuatro de sus dieciséis horas de vigilia en la carretera o reuniendo los recursos para ello, y esta escena no tiene en cuenta el tiempo consumido en otras actividades determinadas por el transporte: el tiempo gastado en hospitales, en juicios por cuestiones de tráfico, en talleres, viendo anuncios de coches o asimilando información sobre el automóvil de sus sueños. En casi todas partes nos encontramos con que el costo total de los accidentes de tráfico se equipara al de las universidades, al tiempo que aumenta con la producción industrial. Pero, aún más revelador es el tiempo que todo ello exige: si se ejerce una actividad profesional, el americano medio invierte mil seiscientas horas anuales para recorrer diez mil kilómetros, lo que representa una media de aproximadamente seis kilómetros por hora que, curiosamente, es la misma velocidad media a la que se desplazan las personas de un país carente de trafico industrializado, con la diferencia de que emplean rutas elegidas libremente y dedicando, no el veintiocho por cien del conjunto del tiempo de que dispone la sociedad, sino el únicamente el 3,8\%. La diferencia entre países ricos y pobres no es que en los primeros la mayoría de la población recorra más millas en una hora de su existencia, sino que los primeros dedican más horas a consumir grandes dosis de energía, desigualmente distribuidas por la industria de la circulación. (Illich, 1974, p.45-46)

Illich evidencia que el estadounidense medio dedica a su automóvil una sorprendente cantidad de dinero, traducible a tiempo de trabajo, para poder comprarlo, llenar su depósito de combustible, pagar las reparaciones, los impuestos, las carreteras y los aparcamientos, y una sorprendente cantidad de tiempo en conducirlo, limpiarlo, gestionarlo y curarse de los accidentes en los que se ve involucrado. Sumando esas dos cifras, el "tiempo directo" dedicado al automóvil y el "tiempo indirecto" dedicado a trabajar para el automóvil, al compararlas con el desplazamiento anual, comprueba que la velocidad (tiempo $/ \mathrm{km}$ recorridos) es poco más alta que la de una persona que camina.
A raíz de este enfoque, se hicieron cálculos similares para otros contextos. En Francia Dupuy \& Robert (1979) se cuestionaban, ¿hace el automóvil ganar tiempo?, y bajo sus cálculos responden que "lejos de ser un instrumento de ganancia de tiempo, el automóvil aparece bajo esta luz como un monstruo cronógrafo" (p.47). Y, como bien señalan Esteban \& Sanz, este término (transporte cronógrafo) es utilizado para describir el fenómeno en el que los esfuerzos requeridos para el funcionamiento del automóvil devoraban su promesa de liberar al hombre del tiempo, llevándole, por el contrario, a acompasar sus resultados globales como mucho a los de la bicicleta. El interés de Dupuy \& Robert, parte por analizar las variaciones de la 'velocidad generalizada' (en el párrafo siguiente se detalla su definición y cálculos), en función del tipo de automóvil y de la categoría social; cabe mencionar que los datos de análisis se refieren al año 1967, mucho antes del alza de los carburantes y cuando no existía limitación de la velocidad. Comprueban que, a modelos determinados, cuanto más se asciende en jerarquía social, mayor es la velocidad generalizada. Esto, sobre todo, por la elevación de la renta, que disminuye el tiempo de trabajo necesario para obtener determinados recursos. Por lo demás, sea cual fuere la categoría social, el modelo de coche más rápido -en términos de velocidad generalizada- es siempre el de mejor jerarquía, que, a su vez, resulta aventajado por la bicicleta.

Se estiman todos los gastos anuales vinculados a la posesión y el uso de un automóvil: amortización de los gastos de adquisición del permiso de conducir, amortización de los gastos de compra del coche; gastos fijos pagables anualmente: patente, seguro, garaje; gastos corrientes de utilización: carburantes, aceite, neumáticos, engrase y limpieza, revisiones periódicas, reparaciones normales o debidas a accidentes, gastos de estacionamiento y peajes, compra de accesorios diversos. Estas erogaciones se convierten en tiempo, dividiéndolas por la renta horaria. El tiempo es, en tal caso, luego, el tiempo que hay que pasar trabajando para obtener los recursos necesarios para la adquisición y utilización del coche. Se lo adiciona al tiempo gastado efectivamente en desplazamientos, y, a este último, se lo estima a partir del kilometraje anual medio, de la distribución de este en tipos de desplazamientos -trayectos domicilio-trabajo, desplazamientos profesionales, vacaciones, desplazamientos privados, recreaciones- del incremento de esta distribución, con una distribución según los tipo de 
velocidad -velocidad en las carreteras, velocidad urbana en las horas de más intenso tránsito y en las horas de menor movimiento, según el tipo de aglomeración-y, por último, de una estimación de las velocidades. Se añaden, por fin, fuera de cuenta, los demás tiempos vinculados a la utilización del coche: tiempo pasado personalmente conversando, tiempo perdido en los embotellamientos, tiempo gastado en la compra de combustibles y accesorios diversos, tiempo transcurrido en el hospital, tiempo perdido en incidentes, etc. El tiempo global así obtenido permite obtener, relacionado con el kilometraje anual, la velocidad general buscada. (Dupuy \& Robert, 1976, p. 47)

Para el caso de España, Naredo \& Sánchez (1974) realizan un análisis de las cuentas del automóvil desde el punto de vista del usuario y no desde el punto de vista de la sociedad. Mencionan que el abaratamiento del automóvil incide favorablemente sobre su eficiencia, al revés que la caída de las velocidades medias, en un periodo de quince años (1974-1991). En 1991, los kilómetros recorridos por cada hora dedicada al automóvil, oscilaban entre 13,8 y 8,8, según los modelos, frente a los 8,7 y 8,0 de 1974. Ello se debía al abaratamiento relativo de los costes del automóvil respecto al salario medio, ya que se habían mantenido invariables las hipótesis de velocidad y recorrido. Añaden, además, que lo que se había modificado sensiblemente, entre 1974 y 1991, es el destino de tiempo que el automóvil exigía del usuario. En 1974, un usuario medio del modelo más barato tenía que dedicarle 3 horas diarias de trabajo para mantenerlo y 1,7 horas de conducción. Sin embargo, en 1991 esta relación se invierte y tendría que destinar 1,2 horas de trabajo y 3 horas de conducción. En un inicio, había que destinarle al automóvil, sobre todo, tiempo de trabajo para pagar sus gastos y amortizaciones. Posteriormente, había que destinarle, en primer lugar, tiempo de conducción para hacer frente a un tráfico más congestionado. También realiza un análisis del transporte colectivo (para el mismo periodo 1974 -1991): comparan el coste del automóvil por kilómetro recorrido, con el billete de metro y autobús. Esta comparación muestra un desplazamiento de los costes, claramente favorables al automóvil privado. Mencionan que estos costes relativamente mayores hacen que la "eficiencia del transporte colectivo dé caída, hasta situarse por debajo de la del automóvil" (p. 9).

Para el caso de Lima, los cálculos se realizarán bajo la metodología y procedimiento de Dupuy \& Robert, y se trabajará con los datos disponibles sobre tráfico y movilidad para el AML, como el "Estudio de tráfico para Lima - 2017", "Encuesta Nacional de Hogares sobre Condiciones de Vida y Pobreza - 2017", "Planos de Estratificación de Lima Metropolitana a nivel de manzana - 2016", del Instituto Nacional de Estadística e Informática - INEI, base de datos de "Lima Cómo Vamos" para el 2017.

Resultados y Discusión sobre el Tiempo Social absorbido por la Movilidad Motorizada en Lima Dupuy \& Robert, al igual que Illych, parten del cálculo del 'costo generalizado', que es la suma de los gastos monetarios vinculados al modo de transporte que se utiliza para un trayecto dado, y de la duración del trayecto convertida en unidades monetarias, por medio de un 'valor del tiempo.' A este valor del tiempo se lo estimaba, en general, igual a la renta horaria del sujeto estudiado. Como dato disponible, tenemos los "gastos anuales", vinculados a la posesión y uso del automóvil, siendo este S. / 12,376.00 según "Estudio de tráfico - 2017" de la Universidad del Pacifico. Este valor incluye costos de adquisición del medio de transporte, teniendo en cuenta la depreciación del producto, el gasto en combustible, los seguros, las reparaciones y los mantenimientos promediados. Al ser el único dato disponible, se tomará para todos los cálculos. Como segundo dato, tenemos el tiempo de desplazamiento promedio anual (602.25 hrs/año). En lugar de convertir los tiempos en gastos, convertiremos el gasto en tiempo. Dividimos los gastos anuales del auto por el 'valor del tiempo' (obtenido a partir del INEI); este tiempo es el que hay que pasar trabajando para obtener los recursos necesarios para la adquisición y utilización del automóvil (1,450.87 hr/año). De la sumatoria de ambos datos obtendremos el "tiempo generalizado" requerido para el uso del automóvil, a partir del cual se obtiene la velocidad generalizada. Cabe mencionar que el tiempo generalizado es la suma del tiempo efectivo de desplazamiento, con el tiempo transcurrido en un trabajo, para obtener los recursos necesarios para el desplazamiento (Dupuy \& Robert, 1976). De la Tabla 1 podemos ver que, para un limeño promedio, el tiempo generalizado requerido es de 2,051.43 horas/año o 5.62 horas/ día, tiempo que requiere trabajar para obtener los recursos necesarios para la adquisición, utilización y desplazamientos diarios en un automóvil.

Al hacer este mismo procedimiento, para conocer las demandas de tiempo para el automóvil, según estrato socioeconómico, se observa que 
las demandas del tiempo para el automóvil se incrementan según estratos. Así, a menor estrado socioeconómico, mayor es el tiempo generalizado para poder acceder a uno, aunque de antemano sabemos que estos no disponen de autos propios y su desplazamiento es mayoritariamente en transporte público. Los estratos bajos necesitarían 6,$87 ; 9,37$; y 13,72 horas (tiempo generalizado), en el caso de que quisieran desplazarse o tener un auto propio. Vale decir que solo trabajarían para acceder y desplazarse en un auto. Los resultados, según estrado socioeconómico, se asemejan a los de Dupuy \& Robert, puesto que, en la medida que los ingresos son mayores, la velocidad generalizada también lo es. Cabe mencionar que los datos obtenidos son básicos, puesto que no se ha considerado desplazamiento privado como vacaciones, actividades recreativas, tiempo perdido en atasco. De tener estos datos y considerarlos, la velocidad generalizada iría disminuyendo.
No tiene caso hallar sólo la velocidad generalizada y el tiempo generalizado para desplazamientos en automóvil privado para el $A M L$, cuando la mayoría de la población se desplaza en transporte colectivo. Partiendo de la encuesta sobre movilidad de Lima Cómo Vamos - 2017, y bajo el mismo procedimiento anterior, hallamos el "tiempo generalizado" de desplazamientos según estrato social para el transporte colectivo. En la Tabla 2 se observa, por ejemplo, para el caso de un limeño del estrato socioeconómico " $\mathrm{C}$ ", el tiempo de desplazamiento promedio es de 0.43 horas diarias. Sin embargo, el tiempo generalizado diario invertido es de 2.68 horas. Este es el tiempo total que esta persona invierte en su desplazamiento, sumado al tiempo de trabajo para poder pagar el costo del mismo. Cabe mencionar que estos cálculos no incluyen el tiempo en los atascos, tráfico o de espera, tiempo que no están considerados en las encuestas.

Tabla 1. Tiempo Generalizado en hr/día para el AML.

\begin{tabular}{|c|c|c|c|c|c|c|c|}
\hline $\begin{array}{l}\text { Gasto anual }{ }^{1} \\
\text { S/. 12,376 (*) }\end{array}$ & $\begin{array}{c}\text { Renta horaria }{ }^{2} \\
\text { (Soles/Hr) }\end{array}$ & $\begin{array}{c}\text { Tiempo } \\
\text { adquisición y } \\
\text { utilización } \\
\text { (Hr/año) }\end{array}$ & $\begin{array}{c}\text { Tiempo } \\
\text { desplazamiento }^{3} \\
\text { (Hr/año) }\end{array}$ & $\begin{array}{c}\text { Tiempo } \\
\text { Generalizado } \\
\text { (Hr/año) }\end{array}$ & $\begin{array}{l}\text { Distancia } \\
\text { recorrida } \\
\text { (Km/año) }\end{array}$ & $\begin{array}{c}\text { Velocidad } \\
\text { Generalizada } \\
(\mathrm{Km} / \mathrm{h})\end{array}$ & $\begin{array}{c}\text { Tiempo } \\
\text { Generalizado } \\
\text { (Hr/día) }\end{array}$ \\
\hline Limeño promedio & 8.54 & $1,450.87$ & 602.25 & $2,051.43$ & 9672.5 & 4.72 & 5.62 \\
\hline \multicolumn{8}{|c|}{ Según Estratos socioeconómicos (2016) } \\
\hline A & 24.39 & 507.42 & 602.25 & $1,109.67$ & 9672.5 & 8.72 & 3.04 \\
\hline B & 10.69 & $1,157.72$ & 602.25 & $1,759.97$ & 9672.5 & 5.50 & 4.82 \\
\hline C & 6.49 & $1,906.93$ & 602.25 & $2,509.18$ & 9672.5 & 3.85 & 6.87 \\
\hline D & 4.39 & $2,819.13$ & 602.25 & $3,421.38$ & 9672.5 & 2.83 & 9.37 \\
\hline $\mathbf{E}$ & 2.81 & $4,404.27$ & 602.25 & $5,006.52$ & 9672.5 & 1.93 & 13.72 \\
\hline
\end{tabular}

Fuente: Elaboración propia con base en INEI, Universidad del Pacifico.

${ }^{1}$ Gasto anual, a partir del "Estudio de tráfico - 2017" realizado por la Universidad del Pacifico; estimaron un gasto semanal para un auto de S. / 238; incluye costos de adquisición del medio de transporte teniendo encuesta la depreciación del producto, gasto en combustible, seguros, reparaciones y mantenimientos promediados.

${ }^{2}$ Renta horaria, a partir de "Encuesta Nacional de Hogares sobre Condiciones de Vida y Pobreza -2017" INEI.

${ }^{3}$ Tiempo de desplazamiento, a partir de "Estudio de tráfico - 2017 (99 minutos tiempo diario promedio para desplazarse al día; en un año hacen 602.25 hrs).

${ }^{4}$ Tiempo Generalizado es la suma del tiempo efectivo de desplazamiento, con el tiempo transcurrido en un trabajo para obtener los recursos necesarios para el desplazamiento.

${ }^{5}$ Distancia recorrida, a partir del "Estudio de tráfico - 2017" promedio de distancia recorrida por limeños para ir a trabajar de 26,8 km., hacen 9,672.5 km en un año.

$(*)$ La moneda del Perú es el "Sol (S/.)", cuya tasa de cambio de la moneda es de US\$1.00=Soles S/.3.33 correspondiente al mes de octubre de 2019.

Tabla 2. Tiempo Generalizado del transporte público.

\begin{tabular}{|c|c|c|c|c|c|c|c|c|}
\hline \multirow[t]{2}{*}{ Estrato } & \multicolumn{2}{|c|}{ Ingreso Mensual Valor del tiempo } & \multirow{2}{*}{$\begin{array}{c}\begin{array}{c}\text { Gasto en } \\
\text { transporte } \\
\text { colectivo }\end{array} \\
\text { Soles/año }\end{array}$} & \multirow{2}{*}{$\begin{array}{c}\text { Tiempo dedicado } \\
\text { al transporte } \\
\text { publico }\end{array}$} & \multicolumn{2}{|c|}{$\begin{array}{c}\text { Tiempo de } \\
\text { desplazamiento }\end{array}$} & \multirow{2}{*}{$\begin{array}{c}\begin{array}{c}\text { Tiempo } \\
\text { generalizado } \\
\text { (Anual) }\end{array} \\
\text { Horas/año }\end{array}$} & \multirow{2}{*}{$\begin{array}{c}\begin{array}{c}\text { Tiempo } \\
\text { generalizado } \\
\text { (diario) }\end{array} \\
\text { Horas/día }\end{array}$} \\
\hline & Soles & Soles/hora & & & $\begin{array}{c}\text { Horas/ } \\
\text { año }\end{array}$ & $\begin{array}{c}\text { Horas/ } \\
\text { día }\end{array}$ & & \\
\hline A & 4570,00 & 22,29 & 6957,08 & 312,07 & 157,71 & 0,50 & 469,79 & 1,51 \\
\hline B & 2507,24 & 12,23 & 5575,96 & 455,90 & 143,83 & 0.46 & 599,74 & 1,92 \\
\hline C & 1318,62 & 6,43 & 4500,08 & 699,60 & 136,13 & 0.43 & 835,74 & 2,68 \\
\hline D & 828,03 & 4,04 & 2923,96 & 723,90 & 129,06 & 0.41 & 852,96 & 2,73 \\
\hline$E$ & 635,71 & 3,10 & 1928,16 & 621,78 & 141,28 & 0.45 & 763,06 & 2,45 \\
\hline
\end{tabular}

Fuente: elaboración propia en base a base de datos de la encuesta sobre movilidad de "Lima como vamos - 2017 " para Lima y Callao. 
La definición que dan Dupuy \& Robert sobre tiempo social, cuando se refieren a la movilidad, es que este se distribuye entre "el tiempo de trabajo y el tiempo de desplazamiento efectivo" (p. 51). En términos generales, es la misma definición que dan sobre el tiempo generalizado. Mencionan, además, que tanto el cálculo de la velocidad generalizada como el tiempo generalizado solo tienen sentido si se satisface cierto número de condiciones. Una de ellas es que este corresponde a un espaciotiempo social determinado. Para el caso de estudio, vendría a ser la sociedad limeña en el contexto actual. Una sociedad cada vez más dependiente de los desplazamientos motorizados, y a medida que disminuye el nivel socioeconómico, el tiempo social absorbido por la movilidad motorizada se incrementa, tanto si se desplazan en auto propio como en transporte colectivo.

\section{Conclusiones}

Se ha evidenciado que el tiempo social que emplea un limeño promedio para desplazarse en automóvil propio es de 5.63 horas/día, tiempo que requiere, no solo para conducir su automóvil sino también el tiempo de trabajo que requiere para su compra y mantenimiento. La velocidad generalizada, a la que va, es de $4.72 \mathrm{~km} /$ hora poco más de la que va una persona caminando $(4 \mathrm{~km} / \mathrm{h})$. Al hacer el análisis por estrato, vemos que a menor estrato socioeconómico mayor es el tiempo generalizado o tiempo social requerido, por lo que, en el caso hipotético que una persona de renta baja quisiera tener un auto, prácticamente trabajaría sólo para este, aunque sabemos que esto no es real, puesto que los estratos socioeconómicos bajos se desplazan mayoritariamente en transporte público.

Para el caso del tiempo social absorbido por el transporte público, se ha evidenciado que, a menor estrato social mayor es el tiempo social requerido. Esto quiere decir que son los estratos socioeconómicos más bajos los mayores afectados por el modelo de movilidad imperante, y tal como menciona Naredo \& Sánchez, la eficiencia del transporte colectivo (para cuestiones de tiempo de desplazamientos) decae hasta situarse por debajo de la del automóvil.

Es claro que el limeño no ve este tiempo invertido en la movilidad como un tiempo perdido. Su percepción tradicional del tiempo ha sido distorsionada. Perdió la libertad de imaginarse a sí mismo en otro papel que no sea el de un usuario dependiente de los desplazamientos motorizados, llegando al punto de identificar el territorio como un paisaje intocable a través del cual se desplaza. De seguir las dinámicas urbanas tal como han sido hasta ahora, cualquier incremento adicional de los tiempos de desplazamientos, sea por las cada vez mayores distancias a recorrer, a causa de la expansión urbana y de la concentración de actividades, o por la ausencia de políticas que busquen fomentar los modos no motorizados. La situación ira empeorando, sobre todo para los estratos socioeconómicos más bajos, quienes no solo tienen todo cada vez más lejos, sino son los que mayor tiempo social invertido en los desplazamientos motorizados tienen, un tiempo que no podrán recuperar.

\section{REFERENCIAS}

Buchanan, C. (1973). El tráfico en las ciudades [Traffic in towns (1961)], Madrid. Editorial Tecnos S.A.

Chión, M. (2002). Dimensión metropolitana de la globalización: Lima a fines del siglo XX. Eure, 28(85), 71-87. Recuperado de https://doi.org/10.4067/S0250-71612002008500005

Dupuy, J.P., y Robert, J. (1979). La traición de la opulencia [La trahison de l'opulence (1976)], Barcelona.Gedisa, S.A.

Estevan, A., y Sanz, A. (1996). Hacia la reconversión ecológica del transporte en España. España, Los Libros de la Catarata. 
Fernández, G., y Vilela, M. (2015). Reflexiones sobre densidad urbana y centralidades en la metrópoli de Lima, siglo XXI. Revista de Arquitectura, Urbanismo y Territorio, pp. 83-114.

González, E., y Del Pozo, J. M. (2012). Lima, una ciudad policéntrica. Un análisis a partir de la localización del empleo. Lima. Investigaciones Regionales, 2012, (23), pp. 29-52

Herce, M. (2009). Sobre la movilidad en la ciudad. España. Editorial Reverté.

Illich, I. (2015). Energía y Equidad, los limites sociales de la velocidad [Énergie et equité (1973)], España. Díaz \& Pons.

Jacob, J. (2011). Muerte y vida de las grandes ciudades [The death and life of great american cities (1961)], España, Capitán Swing Libros, S.L.

Instituto Nacional de Estadística e Informática - INEI. (2017). Encuesta Nacional de Hogares sobre Condiciones de Vida y Pobreza - 2017. Recuperado de https://webinei.inei.gob.pe/anda_inei/index. php/catalog/613/datafile/F2

Instituto Nacional de Estadística e Informática - INEI. (2016). Planos de Estratificación de Lima Metropolitana a Nivel de Manzana 2016. Recuperado de https://www.inei.gob.pe/media/MenuRecursivo/ publicaciones_digitales/Est/Lib1403/index.html

Lima Cómo Vamos. (2017). Encuesta Nacional de Hogares 2017. Recuperado de https://www.limacomovamos. org/data/

Mishan, E.J. (1969). Los costes del desarrollo económico. España, ediciones Orbis, S.A.

Municipalidad Metropolitana de Lima (2014). Plan Metropolitano de Desarrollo Urbano. Lima y Callao 2035. Recuperado de http://comunasurbanas.blogspot.com/2018/09/descarga-plam-2035-planmetropolitano.html

Naredo, J.M., y Sánchez, L. J. (1994). Las cuestas del automóvil desde el punto de vista del usuario. España, Archipielago 1992, Nro 18-19

Vega-Centeno, P. (2017). La dimensión urbana de las centralidades de Lima Norte: cambios y permanencias en la estructura metropolitana. EURE. 2017, 43(129), pp.5-25

Universidad del Pacifico. (2017). Estudio de tráfico - 2017. Recuperado de https://marketwin.pe/estudio-trafico/ 